\title{
A REPRESENTABLE FUNCTOR THEOREM FOR COMPACT ANALYTIC SPACES AND APPLICATIONS
}

\author{
ANDREW J. SOMMESE
}

ABSTRACT. It is shown that the functor given by maps from a given compact analytic space $X$ into elements of a category $C$ of analytic spaces is representable by a compact element of $C$ if and only if $C$ is closed under finite products and the taking of compact subspaces. Various specific choices are considered. Of special interest is the category of compact hyperbolic spaces which gives rise to a birational invariant when applied to projective manifolds.

In this paper I will present a number of new functors and a proposition showing they are representable. Among them the hyperbolic functor is a birational invariant of especial interest. Parts of the below are contained in Appendix III of the author's thesis [5].

The representable functor theorem is a direct generalization of a result of H. Cartan [1].

Lemma I. Let $\left\{X, X_{1}, X_{2}, X_{3}, \ldots\right\}$ be compact analytic spaces. Let $f_{i}: X \rightarrow X_{i}$ and $f_{i+1, i}: X_{i+1} \rightarrow X_{i}$ for all positive integers $i$ be holomorphic surjections such that the diagram

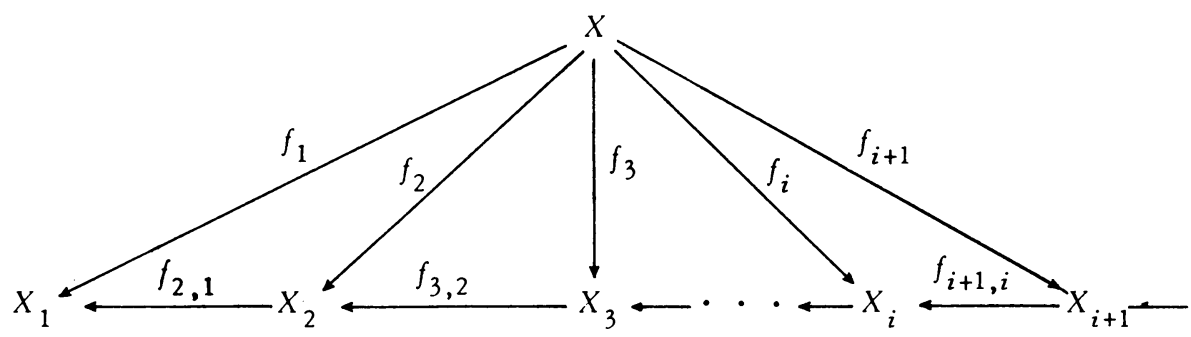

commutes. Then $f_{N+1, N}$ is a biholomorphism for $N \geq n_{0}$ for some integer $n_{0}$.

Remark。Equivalently the category of quotients of a compact analytic space satisfies the ascending chain condition.

Proof。It can be assumed without loss of generality that $X$ and hence the $X_{i}$ are connected. Using the following lemma of Cartan [1, p. 7], it can be assumed that all the $f_{i+1, i}$ are homeomorphisms.

Received by the editors March 25, 1974 and, in revised form, June 27, 1974.

AMS (MOS) subject classifications (1970). Primary 14B99, 32L99, 32C15; Secondary $32 \mathrm{H} 20$.

Key words and phrases. Representable functor, compact complex space, hyperbolic space, complex fibre space. 
Lemma (Cartan). Let $X$ be a reduced analytic space; consider any family of analytic maps $f_{i}: X \rightarrow Y_{i}$ ( $i \in I$, the $Y_{i}$ 's being analytic spaces), and denote by $R_{I}$ the equivalence relation defined on $X$ by these maps. Then for any compact subset $K$ of $X$, there is a finite subset $J$ of $I$ such that $R_{J}$ and $R_{I}$ induce the same relation on $K$.

Let $Y$ be the underlying topological space of the $X_{i}$ and $\theta_{i}$, the structh.re sheaf of $X_{i}$; thus $\left(Y, \theta_{i}\right)=X_{i}$. Now $\theta_{1} \subset \theta_{2} \subset \cdots f_{1} * \theta_{X}$, and all are coherent sheaves over $\theta_{1}$. Therefore by Serre $[4, \mathrm{p} .364]$ all the $\theta_{i}$ are equal for $i$ large enough. Q.E.D.

Theorem I. Let $\mathcal{S}$ be a category of analytic spaces that is closed under finite products and the taking of compact analytic subspaces. Let $X$ be a compact analytic space. Then there exists a compact analytic space $\mathcal{S}(X) \in \mathcal{S}$ and a surjection $f: X \rightarrow \mathcal{S}(X)$ such that given any analytic map $g: X \rightarrow T$ where $T \in \mathcal{S}$, there exists a unique analytic map $h: \mathcal{S}(X) \rightarrow T$ such that the diagram

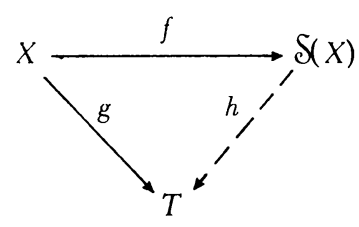

commutes.

Proof. Define $Z_{i}$ an element of $\mathcal{S}$ and $f_{i}: X \rightarrow Z_{i}$ a surjective analytic inductively as follows. Let $Z_{1}$ be an element (possibly a point) of $\mathcal{S}$ such that there exists a surjection $f_{1}: X \rightarrow Z_{1}$. Choose $Z_{i+1}$ if possible such that $f_{i}: X \rightarrow Z_{i}$ factors as $\pi_{i} \circ f_{i+1}$ where $f_{i+1}: X \rightarrow Z_{i+1}$ is a surjection, $Z_{i+1} \in \mathcal{S}$, and $\pi_{i}: Z_{i+1} \rightarrow Z_{i}$ is not a biholomorphism. This gives rise to a commutative diagram:

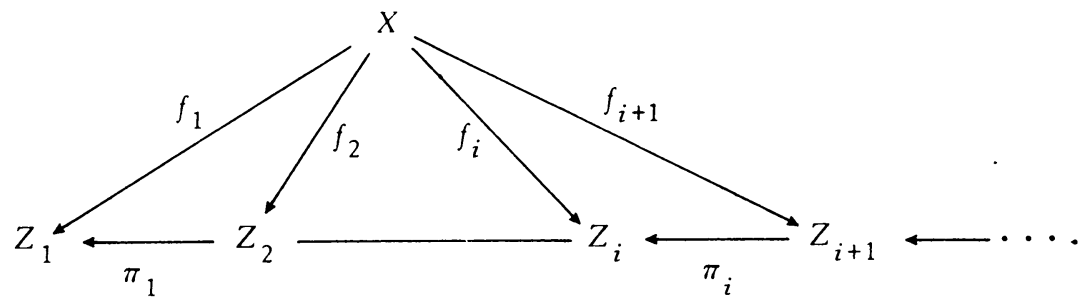

Now by Lemma $I$ the sequence $\left\{Z_{i}\right\}$ terminates, i.e. there is a $Z_{N} \in \mathcal{S}$ and a surjection $f_{N}: X \rightarrow Z_{N}$ and no $Z_{N+1} \in \mathcal{S}$ satisfying the requirements that there exist a surjection $f_{N+1}: X \rightarrow Z_{N+1}$, with $f_{N}=\pi_{N} \circ f_{N+1}$ and $\pi_{N}$ : $Z_{N+1} \rightarrow Z_{N}$ not biholomorphic. $Z_{N}$ is $\mathcal{S}(X)$ and $f_{N}$ is $f$. To see this let $g: X \rightarrow T$ where $T \in \mathcal{S}$. Form the map $A=\left(f_{N}, g\right): X \rightarrow Z_{N} \times T . A: X \rightarrow A(X)$ is a surjection and $A(X) \in \mathcal{S}$ since it is a subspace of a product of elements of $\mathcal{S}$. Now let $p$ denote the projection of $Z_{N} \times T$ onto $Z_{N}$ and let $q$ de- 
note the projection of $Z_{N} \times T$ onto $T$. $p \circ A$ is a factorization of $f_{N}$. Thus by the construction of $Z_{N},\left.p\right|_{A(X)}$ must be a biholomorphism. Finally $b=$ $q\left(\left.p\right|_{A(X)}\right)^{-1}$. This is clear since $h \circ f=q\left(\left.p\right|_{A(X)}\right)^{-1} \circ f_{N}=q A=g$. Q.E.D.

Remark. $\mathcal{S}(X)$ is a retract of the category of compact analytic spaces onto $\mathcal{S}$. The converse of the above theorem is trivially true.

There is one pleasant set of sufficient conditions on $\mathcal{S}$ in order that $\mathcal{S}(X \times Y)=\mathcal{S}(X) \times \mathcal{S}(Y)$. Let $(X, Z)$ be the set of analytic maps from one analytic space $X$ to a second $Z$. Note that if $X$ is compact and reduced, then $(X, Z)$ has a functorial structure as an analytic space whose topology coincides with the compact open topology, and further that $(X \times Y, Z)$ is functorially isomorphic to $(X,(Y, Z))$ where $X$ and $Y$ are compact reduced analytic spaces and $Z$ is an arbitrary analytic space $[2$, Theorems 1,2 , pp. 87, 90]. Note also that the functorial map $f: X \rightarrow \mathcal{S}(X)$ determines a functorial isomorphism $(X, Z) \approx(\mathcal{S}(X), Z)$ for all $Z \in \mathcal{S}$. Conversely $f$ is determined by taking $Z=\mathcal{S}(X)$ and is then the element of $(X, \mathcal{S}(X))$ corresponding to the identity of $(\mathcal{S}(X), \mathcal{S}(X))$. Thus to show that $\mathcal{S}(X \times Y)$ is functorially isomorphic to $\mathcal{S}(X) \times \mathcal{S}(Y)$ it suffices to show that $(\mathcal{S}(X \times Y), Z)$ is functorially isomorphic to $(\mathcal{S}(X) \times \mathcal{S}(Y), Z)$ for every compact $Z \in \mathcal{S}$. Only compact $Z$ are needed because in the above $Z$ must be set equal to $\mathcal{S}(X) \times \mathcal{S}(Y)$ and to $\mathcal{S}(X \times Y)$ which are compact. By functoriality, it follows that $\mathcal{S}(X)$ is reduced if $X$ is.

Corollary I. In addition to the properties of Theorem I, let $\mathcal{S}$ also have the property that $(X, Z) \in \mathcal{S}$ if $X$ and $Z \in \mathcal{S}$ and $X$ is reduced and both are compact. Then $\mathcal{S}(X \times Y)$ is functorially isomorphic to $\mathcal{S}(X) \times \mathcal{S}(Y)$ where $X$ and $Y$ are compact reduced analytic spaces. If $(X, Z)$ is compact whenever $X$ and $Z$ are compact and belong to $\mathcal{S}$ and when $X$ is reduced, and $\mathcal{S}$ is closed under finite to one branched covers, then $(X, Z) \in \mathcal{S}$ and thus the above conclusion holds.

Proof.

$$
\begin{aligned}
(\mathcal{S}(X \times Y), Z) & \approx(X \times Y, Z) \approx(X,(Y, Z)) \approx(X,(\mathcal{S}(Y), Z)) \\
& \approx(\mathcal{S}(X),(\mathcal{S}(Y), Z)) \approx(\mathcal{S}(X) \times S(Y), Z) .
\end{aligned}
$$

The hypothesis of the first sentence of the theorem justifies the fourth isomorphism; the others follow directly from the comments before the statement of the corollary.

For the second assertion observe that by $[2, p .91$, Corollary 1] and by the fact that $(X, Z)$ is compact, there exist $\left\{x_{1}, \ldots, x_{n}\right\} \subseteq X$ such that the map $\Phi(g)=\left(g\left(x_{1}\right), \ldots, g\left(x_{n}\right)\right) \in Z^{n}$, where $g \in(X, Z)$, is an injection $\Phi$ of $(X, Z)$ into $Z^{n}$. Since $Z^{n} \in \mathcal{S}$, it follows that $\Phi((X, Z)) \in \mathcal{S}$ and since $\mathcal{S}$ is closed under finite branched covers and since $\Phi$ is $1-1$, it follows that $(X, Z) \in \mathcal{S}$. Q.E.D. 
Remark. If $(X, Z) \in \mathcal{S}$ for a fixed analytic space $Z$ and for all compact reduced $X$ belonging to $\mathcal{S}$, then $(X, Z) \in \mathcal{S}$ for arbitrary compact reduced $X$ since $(X, Z) \approx(\delta(X), Z)$.

Let $H$ denote the category of compact hyperbolic spaces. By hyperbolic I mean that the Kobayashi pseudo-metric is a metric [3]. This category includes all compact quotients of bounded domains. These are all projective and non-simply connected, and no others members are known, projective or otherwise; there is the obvious conjecture. The functor $H()$ will be called the hyperbolic functor.

Theorem II. If $X$ is a normal compact analytic space, then $H(X)$ is normal and the functorial map $f: X \rightarrow H(X)$ has connected fibres. If $A: X \rightarrow Y$ is a holomorphic surjective map between compact analytic manifolds and $A$ is a biholomorphism between Zariski open sets $E$ of $X$ and $F$ of $Y$, then $H(A): H(X) \rightarrow H(Y)$ is a biholomorphism. Thus $H(X)$ is a birational invariant of $X$ where $X$ is a projective manifold. In particular the concept of a hyperbolic function field, i.e. a function field over $C$ that possesses a unique hyperbolic projective model, is well defined.

Proof. Stein factorize $f: X \rightarrow H(X)$ as $b \circ a$ where $a: X \rightarrow S$ and $b: S \rightarrow$ $H(X)$. By the basic properties of the Stein factorization [5, pp. 1-3] a has connected fibres, $S$ is normal since $X$ is, and $b$ is a finite branched cover with zero dimensional fibres. $H$ is closed under the taking of finite branched covers with zero dimensional fibres $[3$, p. 101] and hence $H$ is also closed under normalization. Therefore $S \in H$, i.e. is hyperbolic, and by the functorial property of $H($ ) shown in Theorem I, $b$ must be a biholomorphism. Thus $H(X)$ is normal and $f: X \rightarrow H(X)$ has connected fibres.

One of the basic properties [3, p. 86] of a compact hyperbolic space $Z$ is that a holomorphic map from a Zariski open set $U$ of $X$ to $Z$ always extends to $X$. Thus there is the commuting diagram:

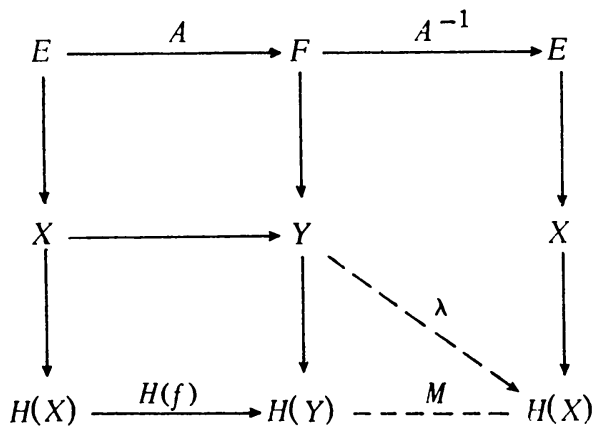

$\lambda$ exists by the above property of compact hyperbolic spaces, and then $M$ exists by functoriality. It is easily seen that $M \circ H(f)=$ identity on $H(X)$. Q.E.D. 
Corollary II. If $X$ and $Y$ are compact reduced analytic space, then $H(X \times Y)=H(X) \times H(Y)$.

Proof。 One could use the second assertion of Corollary I upon observing that $H$ is closed under finite branched covers and further that the basic properties of hyperbolic spaces imply that $(X, Z)$ is compact in the compact open topology if $Z$ is compact hyperbolic and $X$ is a compact analytic space [3].

Instead I will verify the first property. Assume that there were two elements $g$ and $h$ of $(X, Z)$ that had zero distance between them in the Kobayashi pseudo-metric where $X$ and $Z$ are compact hyperbolic and where $X$ is reduced. If $g$ and $h$ are different there exists at least one element $x \in X$ such that $g(x) \neq h(x)$. Now evaluation at $x$ gives a holomorphic map from $(X, Z)$ to $Z$. Since holomorphic maps are distance decreasing with respect to the Kobayashi pseudo-metric, it must follows that $g(x)$ and $h(x)$ have zero distance apart contradicting the assumption that $Z$ was hyperbolic. Q.E.D.

Questions. What property of the function field of a projective manifold corresponds to being hyperbolic? As noted above this purely analytic concept must have a purely algebraic definition!! I know nothing else about the hyperbolic functor. How does $H(X)$ behave when $X$ is deformed? An answer to this might have interesting consequences; $H(X)$ must be a point if $X$ is a Fermat hypersurface, but it is unknown whether $X$, a general hypersurface of high degree in projective space, is hyperbolic.

The category of compact analytic spaces that have some cover biholomorphic to a closed analytic subset of a bounded domain in a Stein space satisfy the hypotheses of Theorem I. By the standard properties of hyperbolic spaces [3], such a compact analytic space is hyperbolic. Denote the functor by $X \rightarrow S(X)$; by the last sentence one gets a factorization $X \rightarrow H(X)$ $\rightarrow S(X)$. The conjecture mentioned before Theorem II is essentially the question whether $H(X) \rightarrow S(X)$ is a biholomorphism.

Another interesting category is the $\mathrm{CP} P^{N}$ spaces for some $N$.

Definition. Any analytic space that is a subspace of a finite product of copies of $\mathrm{CP} P^{N}$ for a fixed $N$ is a $\mathrm{CP} P^{N}$ space.

Let us consider the functor associated to $C P^{1}$ spaces by Theorem I. It is easy to see that one necessary conditions for a $K$ dimensional compact complex manifold to be a $C P^{1}$ space is that the dimension of $H^{r}(X, \mathrm{C})$ be greater than or equal to $\left(\begin{array}{l}K \\ e\end{array}\right)$ where $2 e=r$. Similar conditions exist for the manifold to be a $C P^{N}$ space for any $N$.

I do not know whether the fibre degree of the functorial map from $X$ to $\mathrm{C} P^{N}(X)$ is one or not, or whether normalization takes one out of the $\mathrm{CP} P^{N}$ space category. If the fibre degree is greater than one it is another invariant.

Every $n$ dimensional projective manffold is a $C P^{n}$ space. Any pro- 
jective manifold thus has one very interesting invariant, its dimensional type. By this, I mean the smallest integer $l$ such that it is a $\mathrm{CP}^{l}$ space. In fact there exists a sequence of integers associated to any projective manifold $X$ of dimension $n$. $X$ factors as $X \rightarrow X_{n-1} \rightarrow \ldots X_{1}$ where $X_{i}$ is $\mathbf{C} P^{i}(X)$, the image of $X$ under the factor associated to $C P^{i}$ spaces. Note that $X_{i}=\left(X_{j}\right)_{i}$ for $i \leq j$. This factorization is functorial and thus so are the dimensions. They should be quite calculable.

Another example is the category generated by taking finite products and subspaces of all compact Riemann surfaces of genus greater than or equal to $g$. The case $g=0$ corresponds to $\mathrm{C} P^{1}$ spaces.

The categories of projective analytic spaces and Moisezon spaces also satisfy the hypotheses of Theorem I.

Finally let me comment on the relative case. Assume for simplicity that $S$ is a reduced irreducible normal analytic space that is the union of countably many compact sets. By an analytic space over $S$ one means a pair $(X, g)$ where $X$ is an analytic space and $g$ is a holomorphic map from $X$ to $S$. Given two analytic spaces $A=(X, g)$ and $B=(Y, h)$ over $S$, a $S$ holomorphic map $f: A \rightarrow B$ is a holomorphic map $f: X \rightarrow Y$ such that the diagram

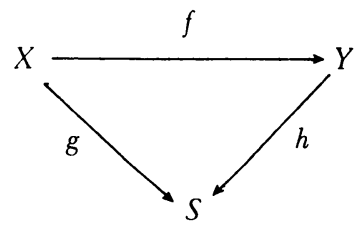

commutes. Cartan's lemma lets one prove a relative version of Lemma I. It says that the category of quotients of an analytic space proper over $S$ satisfies the ascending chain condition over any compact set of $S$. Coupled with the fact that $S$ is a countable union of compact sets one can prove the following version of Theorem I.

Theorem I'. Let $\mathcal{S}$ be a category of analytic spaces satisfying the hypotheses of Theorem I. Let $\mathcal{S}_{S}$ denote the category of all analytic spaces over $S$ such that any fibre belongs to $\mathcal{S}$. Let $X$ be an analytic space proper over $S$. Then there exists an analytic space $\mathcal{S}_{S}(X) \in \mathcal{S}_{S}$ proper over $S$ and an $S$ surjection $f: X \rightarrow \mathcal{S}_{S}(X)$ such that given any $S$ analytic map $g: X \rightarrow T$ where $T \in \mathcal{S}_{S}$, then there exists a unique $S$ analytic map $h:$ $\mathcal{S}_{S}(X) \rightarrow T$ such that the diagram

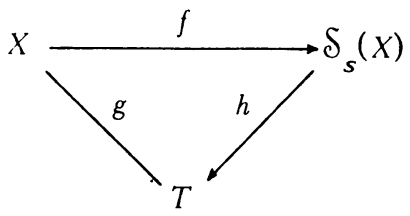


Added in proof. Theorem I also applies to the category of compact complex spaces that have their sheaves of Kaehler differentials spanned by global sections. This functor $X \rightarrow A(X)$ applied to a compact Kaehler manifold has the same image as the Albanese mapping. When applied to nonKaehler compact manifolds the image of the Albanese mapping is generally a quotient space of $A(X)$; for example, when $X$ is the Iwasawa manifold, then so is $A(X)$ and the map $X \rightarrow A(X)$ is the identity mapping.

\section{BIBLIOGRAPHY}

1. H. Cartan, Quotients of complex analytic spaces, Contributions to Function Theory (Internat. Colloq. Function Theory, Bombay, 1960), Tata Institute of Fundamental Research, Bombay, 1960, pp. 1-15. MR 25 \# 3199.

2. A. Douady, Le problème des modules pour les sous-espaces analytiques compacts d'un espace analytique donné, Ann. Inst. Fourier (Grenoble) 16 (1966), fasc. 1, 1-95. MR $34 \# 2940$.

3. S. Kobayashi, Hyperbolic manifolds and holomorphic mappings, Pure and Appl. Math., 2, Dekker, New York, 1970. MR 43 \#3503.

4. J.-P. Serre, Prolongement de faisceaux analytique cohérents, Ann. Inst. Fourier (Grenoble) 16 (1966), fasc. 1. 363-374. MR 35 \#3088.

5. A. J. Sommese, Algebraic properties of the period mapping, Thesis, Princeton University, Princeton, N. J., 1973.

6. K. Stein, On factorization of holomorphic mappings, Proc. Conf. Complex Analysis (Minneapolis, 1964), Springer, Berlin, 1965, pp. 1-7. MR 31 \#2419.

DEP ARTMENT OF MATHEM ATICS, YALE UNIVERSITY, NEW HAVEN, CONNECTICUT 06520

Current address: School of Mathematics, Institute for Advanced Study, Princeton, New Jersey 08540 